Utah State University

DigitalCommons@USU

Mechanical and Aerospace Engineering Student Mechanical and Aerospace Engineering Student Publications and Presentations

$1-10-2019$

\title{
A Sine-Summation Algorithm for the Prediction of Ship Deck Motion
}

Christian R. Bolander

Utah State University

Douglas F. Hunsaker

Utah State University, doug.hunsaker@usu.edu

Follow this and additional works at: https://digitalcommons.usu.edu/mae_stures

Part of the Mechanical Engineering Commons

\section{Recommended Citation}

Bolander, Christian R., and Douglas F. Hunsaker. "A Sine-Summation Algorithm for the Prediction of Ship Deck Motion." OCEANS 2018 MTS/IEEE Charleston. IEEE, 2018.

This Presentation is brought to you for free and open access by the Mechanical and Aerospace Engineering Student Research at DigitalCommons@USU. It has been accepted for inclusion in Mechanical and Aerospace Engineering Student Publications and Presentations by an authorized administrator of DigitalCommons@USU. For more information, please contact digitalcommons@usu.edu.

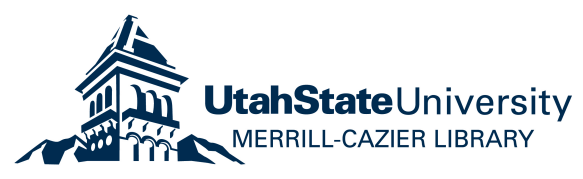




\title{
A Sine-Summation Algorithm for the Prediction of Ship Deck Motion
}

\author{
Christian R. Bolander and Douglas F. Hunsaker \\ Utah State University \\ Department of Mechanical and Aerospace Engineering \\ Logan, Utah U.S.A
}

\begin{abstract}
Landing a fixed-wing aircraft on a moving aircraft carrier is a risky and inefficient process. Having an accurate prediction of ship deck motion decreases the risk posed to both the pilot and the aircraft and increases the efficiency of landing maneuvers. The present work proposes the use of a sine-summation algorithm to predict future ship motion. The algorithm decomposes recorded ship acceleration data into its characteristic harmonic parameters using a fast Fourier transform. The harmonic parameters are then used in a summation of sine waves to create a fit for the acceleration data, which is projected into future time intervals to predict ship motion. An aircraft carrier can supply the prediction made by the algorithm to an autopilot, which then decides to land or make another attempt. Included in this work is a brief overview of ship motion with six degrees of freedom and a description of the method. The results generated by the algorithm are presented for a specific ship motion dataset to provide a point of comparison between the proposed method and other common methods used. The proposed method appears to be accurate in comparison to similar prediction methods, while reducing the computational cost required to make a prediction.
\end{abstract}

\section{NOMENCLATURE}

$\begin{array}{ll}A R & \text { Autoregressive. } \\ A R M A X & \text { Moving average autoregressive. } \\ B F G S & \text { Broyden-Fletcher-Goldfarb-Shanno. } \\ D D G & \text { Guided missile destroyer type vessel. } \\ E L M & \text { Extreme learning machine. } \\ F F T & \text { Fast Fourier transform. } \\ L A M P & \text { Large Amplitude Motions Program. } \\ M C A & \text { Minor component analysis. } \\ N S W C C D & \text { Naval Surface Warfare Center Caderock } \\ & \text { Division. } \\ O N R & \text { Office of Naval Research. } \\ R L S & \text { Recursive least squares. } \\ R M S E & \text { Root mean square error. } \\ S C O N E & \text { Systematic characterization of the naval } \\ W N N & \text { environment. } \\ A & \text { Wavelet neural network. } \\ c & \text { Amplitude. } \\ \hat{c} & \text { Actual value. } \\ f_{N y q u i s t} & \text { Predicted value. } \\ f_{s} & \text { Nyquist frequency. } \\ \mathbf{F}_{S} & \text { Sampling frequency. } \\ \mathbf{I} & \text { Net surface force vector. } \\ m & \text { Inertia tensor. } \\ \mathbf{M}_{S} & \text { Mass. } \\ & \text { Net moment vector about the body-fixed } \\ & \text { origin. }\end{array}$

$\begin{array}{ll}N & \text { Total number of data points. } \\ P_{x x} & \text { Power spectral density. } \\ S_{A} & \text { Amplitude spectrum. } \\ S_{P} & \text { Phase spectrum. } \\ t & \text { Time. } \\ T_{\min } & \text { Shortest wave period. } \\ \mathbf{V} & \text { Translational velocity vector. } \\ \mathbf{W} & \text { Weight vector. } \\ X & \text { Ship deck surge coordinate. } \\ Y & \text { Ship deck sway coordinate. } \\ Z & \text { Ship deck heave coordinate. } \\ z & \text { Constant offset. } \\ \Delta t & \text { Time sample interval. } \\ \delta t & \text { Time step. } \\ \theta & \text { Ship deck pitch coordinate. } \\ \lambda & \text { Damping coefficient. } \\ \phi & \text { Ship deck roll coordinate. } \\ \phi_{0} & \text { Phase shift. } \\ \psi & \text { Ship deck yaw coordinate. } \\ \omega & \text { Frequency. } \\ \omega_{\max } & \text { Highest frequency used in the sine- } \\ \omega & \text { summation. } \\ & \text { Rotational velocity vector. }\end{array}$

\section{INTRODUCTION}

The prediction of ship deck motion is of interest to military pilots due to the difficulty of landing aircraft on a moving ship deck [1] [2]. In making a landing attempt, the pilot guides the aircraft in-line with the aircraft carrier. As the aircraft descends to contact the carrier, the motion of the ship deck can quickly change the distance between the deck and the landing gear of the aircraft, causing premature engagement of the landing gear or decreasing the amount of runway available to the pilot.

The consequences of an abrupt landing of the aircraft can be significant. The sudden motion of the ship deck can cause increased wear to landing gear, necessitate multiple landing attempts, and even result in the loss of an aircraft. In addition to the material costs of such a landing, this situation also poses a safety risk to pilots. An accurate prediction of the motion of the ship would mitigate some of the risk associated with landing on an aircraft carrier. A pilot would be able to avoid an undesirable landing and increase the efficiency of landing maneuvers by knowing if the landing is favorable in advance. Understanding the landing conditions requires an 
accurate prediction of how the aircraft carrier will move in the future.

The challenge with making such predictions comes from what can appear to be random ship deck movement [3] [4]. Ship deck motion is a combination of two phenomena: the harmonic forces generated by ocean waves and the dynamic response of the ship [5]. These phenomena combine to produce the final motion of the ship deck.

Ocean waves are divided into two main categories: sea and swell [5]. Sea is defined as a train of waves driven mostly by the local wind conditions. Swell refers to the remnants of a wave that has propagated out of the area where it was generated [5]. Swell has longer wavelengths and a more predictable height than sea. Surface waves are a combination of both sea and swell, both of which are in constant flux, and therefore can potentially consist of an infinite number of harmonic waves.

The contribution of the dynamic response of the ship to the resultant ship deck motion is derived explicitly by Journee and Pinkster's work [6]. A ship structure contributes its own dynamic components to the ocean wave, the combination of which creates the resultant waveform measured by sensors on the ship as shown in Fig. 1 and Fig. 2. Though the motion caused by the combination of the response of the ship and ocean can be complex, the total response can be expressed as a summation of sine waves with various coordinate directions, amplitudes, phase angles, and frequencies [2] [3].

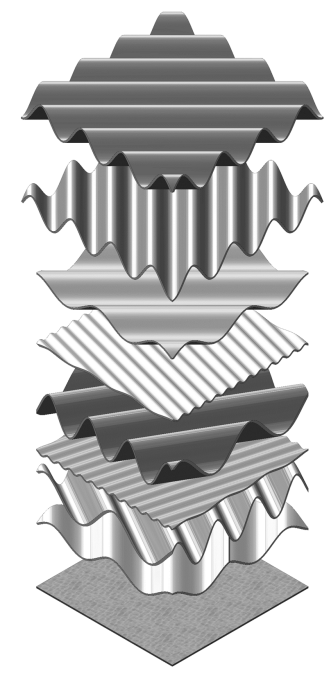

Fig. 1. Three-dimensional representation of ship deck motion.

Many approaches have been taken to predict ship deck motion. In the past, a great deal of emphasis was placed on using time-series analysis to predict ship motion. Such methods include autoregressive (AR) [7] [8] [9] and moving average autoregressive (ARMAX) [8], as well as Kalman filters [4] [9] [10] [11] [12], Wiener filters [7] [13], and the Volterra model [10]. Time-series analyses are still used commonly today; however, most modern approaches utilize

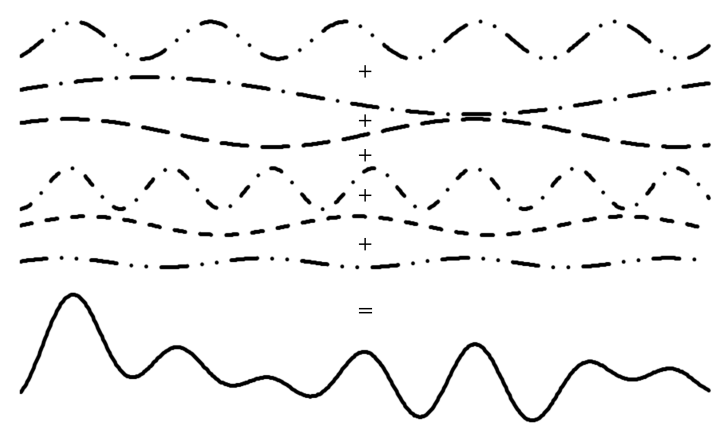

Fig. 2. Two-dimensional representation of ship deck motion.

neural networks to make predictions. Some of the methods that use neural networks include minor component analysis (MCA) [7] [14] [15] [16], extreme learning machines (ELM) [17] [18], and the wavelet neural network (WNN) [19]. The results of many of these methods will be compared to the proposed method, which employs a form of time-series forecasting, in Section IV.

This paper outlines the use of a sine-summation method to predict ship motion. The algorithm fits a summation of sine waves to original dataset and projects the summation into future time intervals. Acceleration data collected aboard current aircraft carriers can be used in conjunction with the proposed method to make predictions onboard the vessel. The prediction can then be transmitted to the aircraft in advance of a landing attempt to indicate the suitability of landing within the projected time frame. An autopilot could then use the prediction based on original dataset to determine if an ideal landing speed and descent trajectory exists in the next several seconds, and the decision could be made to touch down immediately or make another attempt later.

\section{Ship Motion}

An understanding of the basic physics of ship deck motion is fundamental to the concepts employed in the proposed prediction method. A ship is a system with at least six degrees of freedom, including translational and rotational components as shown in Fig. 3. Here we use a coordinate system such that the $\mathrm{X}$-axis points out the front, or bow of the ship, the Y-axis points out the left, or port side of the ship, and the Z-axis points out the top of the ship. Movement in the translational (X, Y, and $\mathrm{Z}$ ) directions are defined as surge, sway, and heave respectively. Rotation about the $X$, $\mathrm{Y}$, and $\mathrm{Z}$ axes are defined as roll, $\phi$, pitch, $\theta$, and yaw, $\psi$, respectively. The positive directions of rotation are defined via the right-hand rule as shown in Fig. 3.

In terms of the body-fixed coordinate system shown in Fig. 3 , and assuming the ship motion can be described with rigidbody displacement, Newton's second law can be written in vector notation as

$$
\mathbf{F}_{S}+\mathbf{W}=\frac{d}{d t}(m \mathbf{V})+\boldsymbol{\omega} \times(m \mathbf{V})
$$




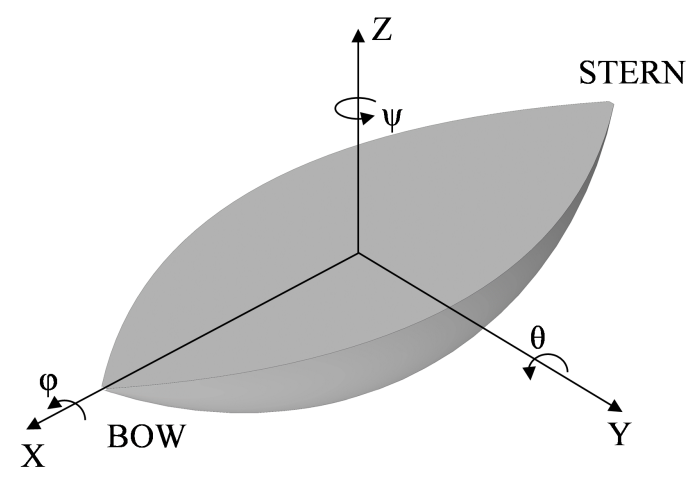

Fig. 3. Accepted ship motion model with six degrees of freedom.

and

$$
\mathbf{M}_{S}=\frac{d}{d t}([\mathbf{I}] \boldsymbol{\omega})+\boldsymbol{\omega} \times([\mathbf{I}] \boldsymbol{\omega}) .
$$

The translational, rigid-body motion of the ship is governed by (1) and the rotational, rigid-body motion of the ship is governed by (2). These two equations together express the complete rigid-body motion of the ship in all six degrees of freedom.

Each degree of freedom in ship motion can be characterized as a spring-mass-damper system subject to a harmonic forcing function [3] [13]. For any arbitrary degree of freedom $x$, the differential equation of motion for a spring-massdamper can be written as

$$
m \frac{d^{2} x}{d t^{2}}+c \frac{d x}{d t}+k x=F \sin (\omega t)
$$

if the harmonic forcing function is composed of a single sine wave.

Due to the coupling between the degrees of freedom in the system, (3) does not explicitly characterize each degree of freedom individually. The coupling effects between each degree of freedom can be considered part of the forcing function on the right-hand side of (3) or as a disturbance to the system.

Solving (3) for the position as a function of time and assuming that the elastic modes of the ship contribute little to the motion of the ship, yields

$$
x(t)=C_{1} e^{\lambda_{1} t}+C_{2} e^{\lambda_{2} t}+A_{f} \frac{F}{k} \sin \left(\omega t-\phi_{0}\right) .
$$

The first two terms on the right-hand side correspond to the motion of the ship governed by its own dynamic response. The last term is the contribution of the ocean waves and coupling effects from other degrees of freedom to the resultant ship deck motion.

The dynamic response of the ship contained in the first two terms can be solved as a general eigenproblem. A purely real eigenvalue is not oscillatory and yields an exponential motion that will converge to equilibrium if negative and diverge if positive. A complex pair of eigenvalues yields oscillatory motion where damping is based on the real component of the eigenvalue. The resultant motion will be damped if the real component is negative, undamped if it is zero, and divergent if it is positive. If a purely real eigenvalue is equal to zero, the motion is described as simple rigid-body displacement.

While the eigenvalues give information on damping and frequency, the resultant components of the eigenvectors give the relative amplitudes and phase shifts contributed by each degree of freedom. Using the information given by the eigenvalues and eigenvectors, the first two terms in (4) fully define the dynamic response of the ship when subjected to a forcing function.

The last term in (4) describes the contribution of the harmonic force of the ocean and coupling effects on the resultant ship motion. As both the dynamic response of the ship and the forcing function of the ocean can be harmonic, they can be described as a summation of sine waves [3] [5] [20]. More information on the physical significance of each term in (4) can be found in the theory of ship hydromechanics [5].

The underlying idea emphasized in this brief overview of the physics of ship motion is the nature of each term in (4). The first two terms describe the ship's dynamic contributions to the resultant ship motion, while the last term describes the contribution of the harmonic force of the ocean waves. Assuming that the dynamic motion of the ship is oscillatory or that the forcing function is much greater than the first two terms, the resultant motion can be represented by a combination of the dynamic motion of the ship and the forcing function of the ocean.

\section{Sine-Summation Prediction Method}

As stated in Section II, ship motion can be approximated by a summation of harmonic waves. A simple, harmonic wave is made up of five components: amplitude, frequency, phase shift, damping coefficient, and constant offset, which can be written as

$$
A e^{-\lambda t} \sin \left(\omega t+\phi_{0}\right)+z .
$$

Due to the relatively small effect that damping has over a short period of time on a large, slow-moving vessel such as a guided missile destroyer (DDG), the damping factor can be ignored [3] [5] and (5) can be rewritten as

$$
A \sin \left(\omega t+\phi_{0}\right)+z
$$

If an infinite number of sine waves were used, the past ship motion data could be described exactly; however, at some point the number of sine waves must be truncated. The proposed method constructs a sine wave summation iteratively by adding a single sine wave per iteration and minimizing the root mean square error (RMSE) between the past ship motion data and the sine summation approximation. There are two distinct components present in the method: a start-up procedure and a real-time algorithm. The proposed method requires a dataset containing time and motion data for a single degree of freedom and the range of data over which the fit will be made. What follows is a description of the start-up procedure of the proposed prediction method. 


\section{Step I. Determining Harmonic Components}

Before outlining the steps used in the proposed algorithm, it is necessary to establish a clear nomenclature for the data being used. There are four datasets that will be referred to throughout the rest of this work: these are the original dataset (or simply the dataset), the actual ship motion data, the prediction, and the sine-summation fit. The original dataset and the actual ship motion data are both found using the ship's sensors. The original data is data that the ship has gathered in the past, and the actual motion is that which is obtained after the algorithm starts. The sine-summation fit is a fit of the original dataset made by the algorithm, while the prediction represents the prediction of the actual ship motion generated by the algorithm.

The first iteration of the method attempts to fit the original dataset using a single sine wave. To accomplish this, approximations for an amplitude, frequency, phase angle, and constant offset are needed. A fast Fourier transform (FFT) is performed on the dataset, which divides the acceleration data into its frequency components according to their influence. The power spectrum is then calculated as

$$
P_{x x}=\left|F F T^{2}\right| \delta t^{2},
$$

where $\delta t$ is the time step in the dataset [21]. In addition to frequency information, the output of the FFT is used to find information about the relative amplitude and phase shift associated with each frequency component. This is accomplished using

$$
S_{A}=\frac{\sqrt{2} \sqrt{\operatorname{real}[F F T]^{2}+\operatorname{imag}[F F T]^{2}}}{N}
$$

for the amplitude spectrum, and

$$
S_{P}=\arctan \left(\frac{\operatorname{imag}[F F T]}{\operatorname{real}[F F T]}\right)
$$

for the phase spectrum [21]. Each spectrum is found over the same frequency domain, and can therefore link the most influential frequency with a corresponding amplitude and phase angle. The phase angles given by the phase spectrum represent the phase shift relative to the start of the time domain, and take values from $-\pi$ to $\pi$.

The most influential frequency is chosen along with the corresponding amplitude and phase shift to be used as initial guesses for the harmonic parameters of the first sine wave. The amplitude spectrum for the original dataset, which was found using the FFT, contains only positive values. Due to the inclusion of a phase shift, $\phi_{0}$, there is no need to accommodate a negative amplitude, as the wave can simply be shifted accordingly. An estimate for the constant offset is determined by taking the average value of the dataset being analyzed.

\section{Step II. Down-sample the Dataset}

To reduce the computational complexity of the optimization that will be described in Step III, the original dataset is down-sampled using the shortest wave period as a benchmark. The shortest wave period can be found by extracting

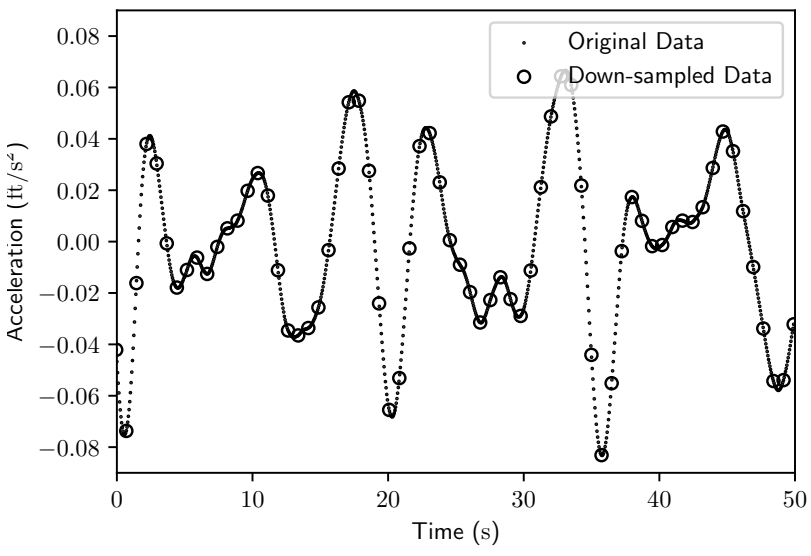

Fig. 4. Effect of down-sampling with four data points per minimum period on a dataset.

from the power spectrum the highest frequency component the will be used in the sine-summation fit and calculating the period using

$$
T_{\min }=\frac{2 \pi}{\omega_{\max }} .
$$

If the number of sine waves that will be used to create the sine-summation fit is given as $n$, then the set of highest $n$ frequencies will be found according to their influence, and the highest frequency (i.e. that with the lowest influence) from that set will be taken as $\omega_{\max }$. The algorithm may also be run until adding another sine wave to the sinesummation fit doesn't appreciably change the RMSE of the fit. In this case, the Nyquist frequency can be calculated using the sampling frequency, $f_{s}$, with

$$
f_{\text {Nyquist }}=\frac{f_{s}}{2} \text {. }
$$

The Nyquist frequency can then be taken as $\omega_{\max }$ after being converted to units of $\frac{\mathrm{rad}}{\mathrm{s}}$.

The dataset is down-sampled by taking four data points for every minimum period as shown in Fig. 4. This allows the frequency components present in the dataset to be preserved while improving the speed of the algorithm and cutting down on computational time. Taking four data points provides a conservative approach to down-sampling, since theoretically only two data points are needed for every minimum period. Four data points are kept to ensure that even high frequency datasets are accurately characterized after down-sampling.

\section{Step III. RMSE Optimization of the Sine Summation}

The optimization of the sine wave is accomplished by adjusting the harmonic parameters in (6) to minimize the RMSE. The RMSE is calculated as

$$
\operatorname{RMSE}=\sqrt{\frac{\sum_{1}^{N}(\hat{c}-c)^{2}}{N}},
$$

where $\hat{c}$ represents the value of the sine-summation fit and $c$ represents the value of the dataset. The Broyden- 
Fletcher-Goldfarb-Shanno (BFGS) [22] [23] [24] [25] gradient descent method is used to minimize the RMSE. Other optimization routines were available in the Python libraries used, including the Nelder-Mead Simplex method [26], the Newton-Conjugate-Gradient algorithm, and the Least Squares method.

The Simplex algorithm was the simplest method, but increased the computational time significantly. The NewtonConjugate-Gradient algorithm and the Least Squares method require a Jacobian or Hessian to optimize the RMSE, which is very costly to perform in subsequent iterations when many parameters need to be optimized. Due to the increased computational complexity of these other methods, the BFGS method was determined to be an appropriate optimization method for this application. The optimization routines described are readily accessible in existing Python libraries, but other options may be available depending on the language.

\section{Step IV. Sum the Sine Waves and Calculate the Fit Error}

The optimized harmonic parameters found in Step III were stored so that they could be used as the initial guesses for the next iteration of the start-up procedure. As additional sine waves are added to the sine-summation fit, they are summed together to form the fit of the original dataset.

The optimized sine wave from the first iteration is subtracted from the original dataset to find the error of the fit, which is used by the FFT in the next iteration. The fit error retains all the frequency information of the original dataset, excluding the frequencies that have been extracted. Hence, future iterations of the start-up procedure have to analyze only the most prominent frequencies that have not been previously analyzed.

While the initial guesses for the harmonic parameters are obtained using an FFT on the fit error of the previous iteration, the optimization minimizes the RMSE between the fit and the original dataset, not the fit error. Each iteration adds three additional harmonic parameters (composing a full sine wave without a constant offset) to be optimized. The harmonic parameters from previous iterations were already optimized, so they change very little in future iterations.

Steps I through IV can be repeated until the RMSE between the sine-summation fit and the original dataset change by less than a threshold value or until a predetermined number of sine waves had been generated. The final form of the sine-summation approximation is

$$
f(t)=\sum_{n=1}^{N} A_{n} \sin \left(\omega_{n}+\phi_{0_{n}}\right)+z .
$$

Figure 5 shows an example of Steps I - IV of the start-up procedure progressing through three iterations. If only one sine wave is used to fit the original data shown in Fig. 5, the resultant RMSE would be $0.464 \frac{\mathrm{ft}}{\mathrm{s}^{2}}$. With two sine waves, the RMSE falls to $0.363 \frac{f t}{s^{2}}$ and with three sine waves the RMSE is $0.280 \frac{f t}{s^{2}}$. As more sine waves are added, a better approximation of the original dataset is made. This can be seen in Fig. 6, where 14 sine waves were used in the fit.

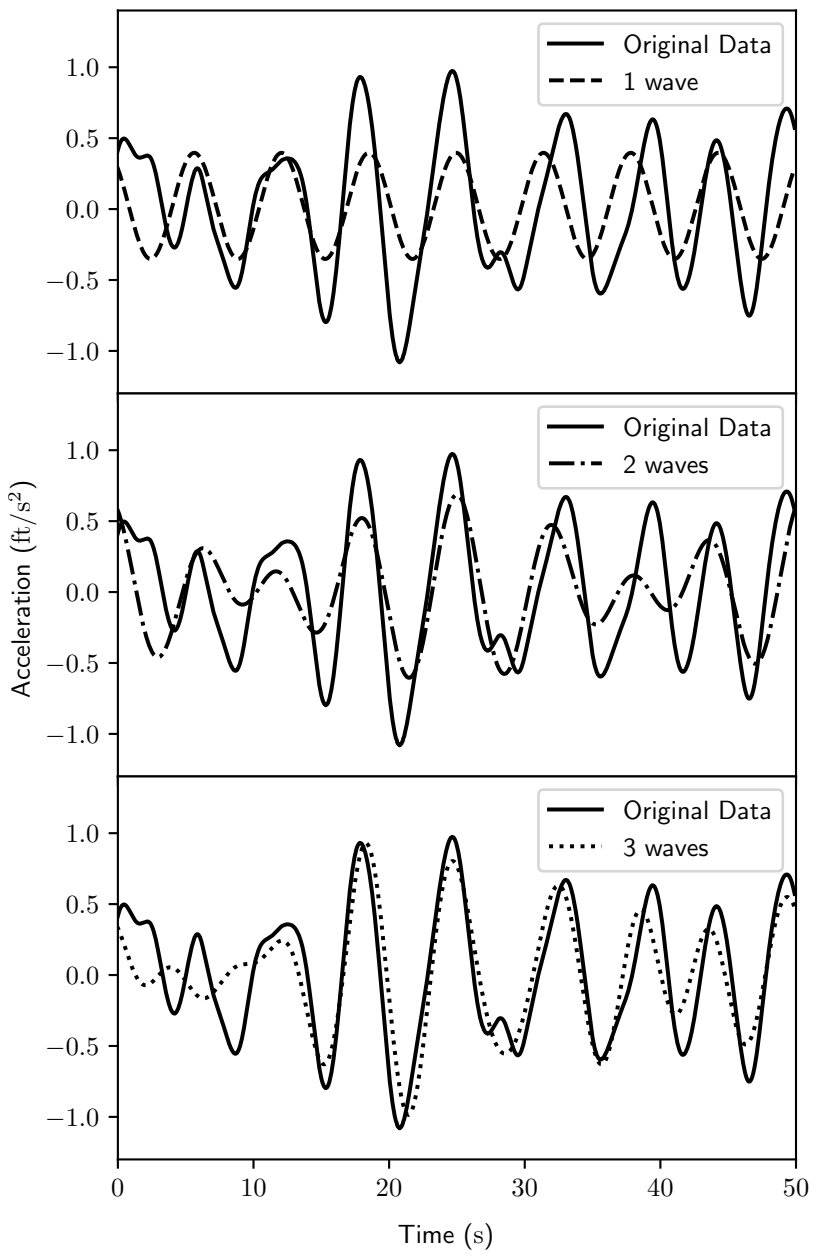

Fig. 5. The sine-summation process using three sine waves to fit a dataset.

\section{Step V. Project the Sine Summation}

Once the given number of sine waves has been generated using Steps I through IV, the sine-summation fit is projected into the future by some time interval. This prediction is then compared to the actual ship motion to compute the RMSE. The RMSE of the prediction is reported in both dimensional and nondimensional forms. The dimensional form of the RMSE is used to more easily make comparisons between different prediction methods, which often express results in terms of a dimensional RMSE value. The nondimensional RMSE normalizes the RMSE values for more accurate comparisons when dealing with high-amplitude datasets. The proposed algorithm normalizes the prediction RMSE by the maximum value in the original dataset to nondimensionalize the RMSE. 


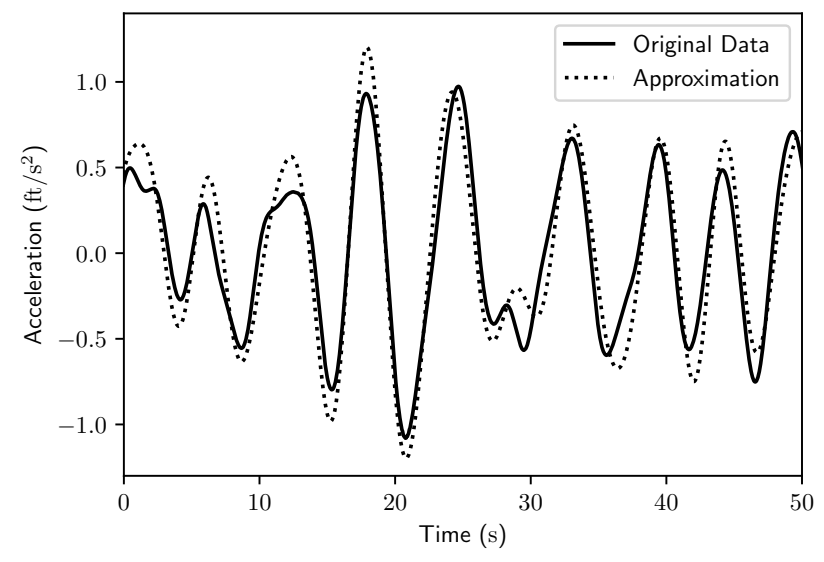

Fig. 6. The final sine-summation fit of the original data using 14 sine waves.

\section{Real-Time Implementation}

As mentioned previously, real-time capabilities have been developed for the proposed algorithm. After running the start-up procedure with a given number of sine waves over a specific dataset, the final amplitudes, frequencies, phase shifts and offset are stored along with the amount of time taken to perform the analysis. This computational time is then used to shift the original dataset, which allows the algorithm to stay updated with the data collected in real-time by the sensors on the ship.

After shifting the data, Step III is performed again with all the harmonic parameters from the first analysis used as initial guesses. The maximum number of iterations performed by the optimization routine is drastically reduced during the analysis over the shifted dataset, since relatively few data points are added to the dataset. In a practical case, the startup procedure could be initiated several minutes before the aircraft attempts to land and transition into the real-time updates to provide more timely predictions for the pilot.

\section{EXAmple Results}

The proposed algorithm was tested using the systematic characterization of the naval environment (SCONE) data produced by Alan Schwartz under funding from the Office of Naval Research (ONR) [27]. The SCONE dataset represents surface combatant and carrier hullforms, specifically a DDG51 type ship. Simulated deck motion from the Large Amplitude Motions Program (LAMP) code [28] is tabulated for all six degrees of freedom in ship deck motion. Each degree of freedom contains information on the displacement, velocity and acceleration of the ship. The SCONE datasets are also characterized by the magnitude of heave and roll rates, with "low", "moderate", and "high" specifications, representing varying sea states that a ship may experience.

For each heave or roll rate specification, five simulations were recorded with random wave phases. Each dataset contains a 30-minute time history at a sampling rate of $20 \mathrm{~Hz}$. The predictions simulate over the time-domain and

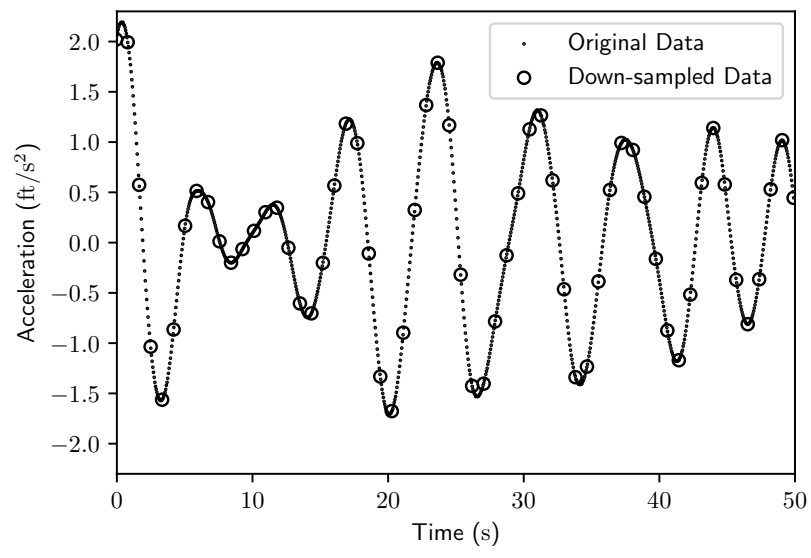

Fig. 7. Heave data down-sampled at four points per minimum period.

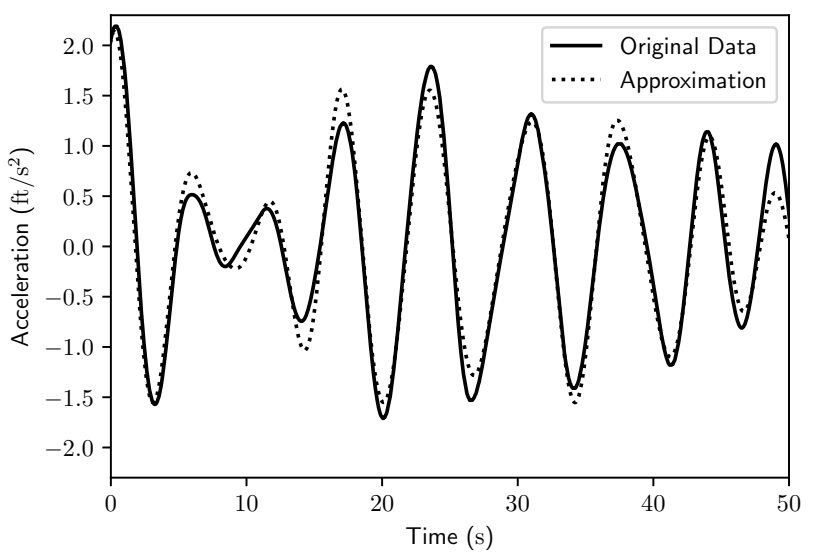

Fig. 8. The sine-summation fit of the heave dataset using five sine waves.

incorporate wave forcing and hydrostatic restoring forces. The SCONE dataset is open to the public, and any interested readers should refer to the Acknowledgments Section for distribution information.

An example of the proposed algorithm applied to a specific dataset will be explored in this section by first showing the start-up procedure. Heave data from the first wave phase specification of a low amplitude SCONE dataset was used. A sampling interval of $50 \mathrm{~s}$ was used with a prediction interval of $10 \mathrm{~s}$. The down-sampled dataset, sine-summation fit, and prediction are shown in Fig. 7, Fig. 8, and Fig. 9 respectively. The nondimensional RMSE for this prediction was 0.660 and it took $0.88 \mathrm{~s}$ to generate the prediction using the start-up procedure.

The RMSE of any prediction is a multi-dimensional optimization problem that depends on the application. Several of these dimensions will be explored here. First, the nondimensional RMSE for the sine-summation fit was recorded as a function of the number of sine waves used. This data is shown in Fig. 10 for different sampling intervals, $\Delta t$, from $50 \mathrm{~s}$ to $125 \mathrm{~s}$. It can be seen that the general trend of the fit 


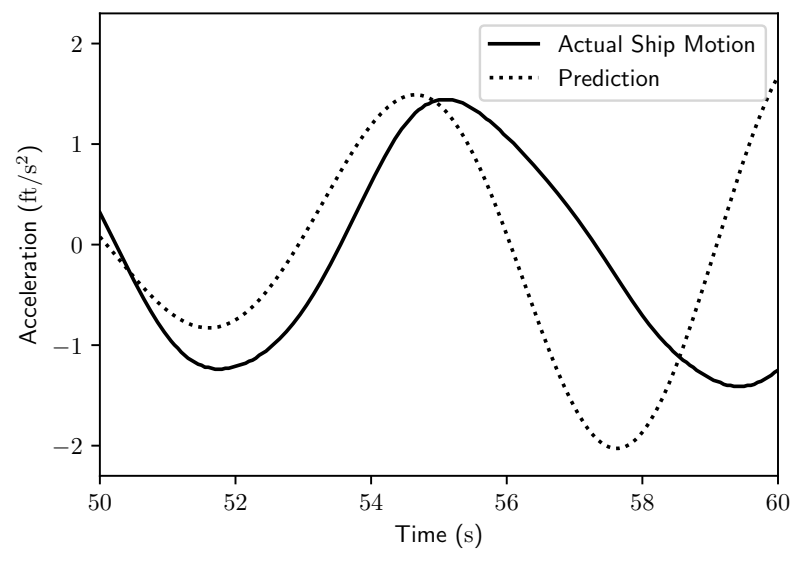

Fig. 9. Motion prediction over $10 \mathrm{~s}$ for the heave dataset.

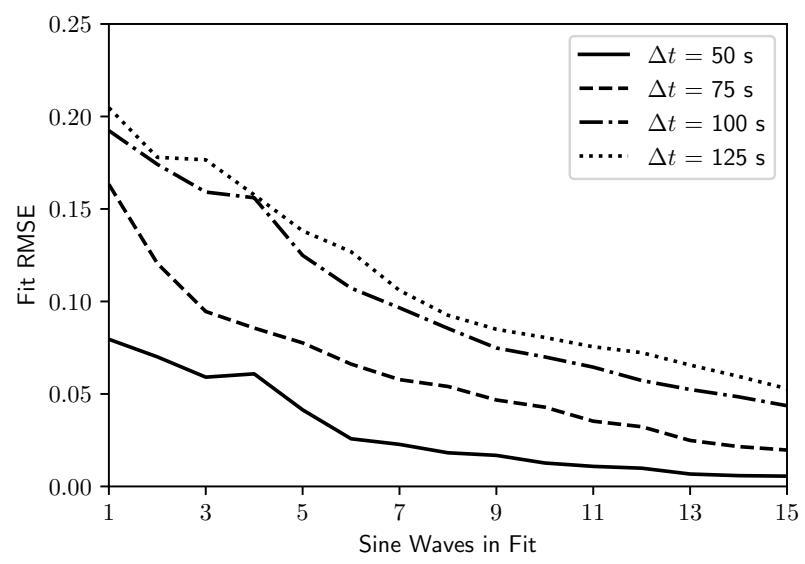

Fig. 10. The fit RMSE as a function of the number of sine waves used in the fit for various sampling intervals.

RMSE in Fig. 10 indicates that increasing the number of sine waves in the sine-summation fit reduces the RMSE, thereby providing a better approximation of the original data.

Keeping the number of sine waves constant at 10, Fig. 11 shows the nondimensional RMSE as a function of the length of the prediction interval. As the prediction interval increases, the prediction RMSE value also increases. This indicates that the algorithm's ability to accurately predict the ship motion decreases the farther into the future that the prediction is made. Figure 11 also indicates that the prediction RMSE is lower for the smaller sampling interval than for the larger intervals. This is likely because the data closer to real-time has more of an impact on the motion of the ship than the older data.

Table I shows the minimum dimensional prediction RMSE for each of the six degrees of freedom using a sampling interval of $50 \mathrm{~s}$ of original data and a $15 \mathrm{~s}$ prediction interval. The number of sine waves used to make the sine-summation fit and the corresponding nondimensional RMSE values are also included. In each of these cases up to 10 sine waves

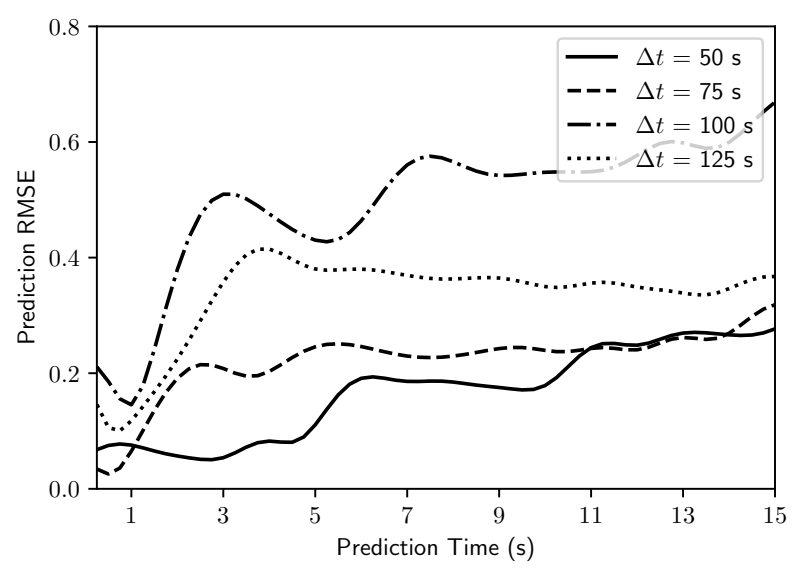

Fig. 11. RMSE as a function of prediction time for various sampling intervals.

were allowed in the fit, and the prediction RMSE was taken for each case.

TABLE I

Minimum PREDiction RMSE

\begin{tabular}{|c|c|c|c|}
\hline & $\begin{array}{c}\text { Sine } \\
\text { Waves }\end{array}$ & $\begin{array}{c}\text { Dimensional } \\
\text { RMSE }\end{array}$ & $\begin{array}{c}\text { Nondimensional } \\
\text { RMSE }\end{array}$ \\
\hline Surge & 6 & $0.07731 \mathrm{ft} / \mathrm{s}^{2}$ & 0.46856 \\
\hline Sway & 4 & $0.35107 \mathrm{ft} / \mathrm{s}^{2}$ & 0.36044 \\
\hline Heave & 5 & $0.92698 \mathrm{ft} / \mathrm{s}^{2}$ & 0.42328 \\
\hline Roll & 4 & $0.31880 \mathrm{deg} / \mathrm{s}^{2}$ & 0.33522 \\
\hline Pitch & 5 & $0.33103 \mathrm{deg} / \mathrm{s}^{2}$ & 0.57471 \\
\hline Yaw & 2 & $0.06950 \mathrm{deg} / \mathrm{s}^{2}$ & 0.40407 \\
\hline
\end{tabular}

As shown in Table I, the number of sine waves used to make the best prediction with this specific dataset is no greater than 6 . This is likely due to the relatively short sampling interval over which the sine-summation fit was made. In addition, while the dimensional RMSE values in Table I may indicate a better prediction for one dataset over another, the nondimensional RMSE values show that each of the predictions have very similar error values.

The proposed method has prediction errors of similar magnitude as the results of other methods. No information was found on the datasets were being used by each of the other methods, meaning a direct comparison is impossible; however, the trends examined are still valuable. It should be mentioned that since the reported errors are dimensional RMSE values, the dataset on which the prediction is being made will affect these comparisons greatly.

Zhao calculates a minimum RMSE of $0.0538 \mathrm{~m}$ for a 5 $s$ prediction using an MCA algorithm [7]. Over the same dataset and time interval, Zhao reported an RMSE of 0.1207 $\mathrm{m}$ using an AR algorithm, $0.1248 \mathrm{~m}$ using the Wiener prediction method, and $0.5287 \mathrm{~m}$ using a three-layer neural network. The hybrid AR method produced an RMSE of just under 0.15 deg on pitch data with a 5 s prediction.

In Yang's work, a recursive least squares (RLS) method 


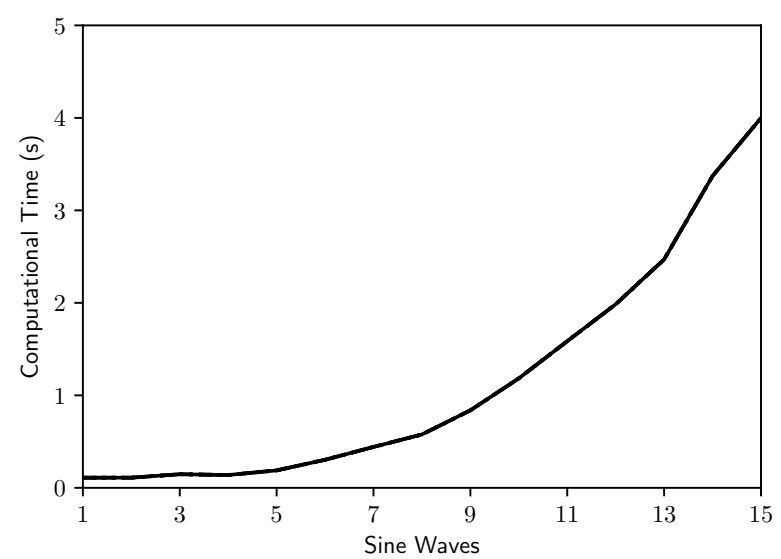

Fig. 12. Computation time for the start-up procedure using a $50 \mathrm{~s}$ sampling interval on the original data.

was employed to make 5 and $10 \mathrm{~s}$ predictions using pitch data [12]. The RLS method had an RMSE of $0.06548 \mathrm{deg}$ for the $5 \mathrm{~s}$ prediction and $0.1339 \mathrm{deg}$ for the $10 \mathrm{~s}$ prediction. Utilizing a WNN, Horn generated an RMSE of 0.3727 deg for a $3 \mathrm{~s}$ prediction for the yaw direction [14].

Keke [8] and Xiuyan [10] used an ARMAX and a combination forecasting method [29] [30] respectively to make their predictions. The results were presented using a mean absolute percentage error. This form of error takes the absolute value of the error and normalizes by the actual value. This artificially inflates the error values when the dataset crosses zero, making it impractical to use with the given SCONE dataset.

When compared to the results in Table I, the other methods seem to generate similar RMSE values as the proposed method. It is difficult to accurately compare the results from these different methods, since the RMSE is dependent on fitting a specific dataset and the dataset in the references discussed are not explicitly stated; however, the general magnitudes of the RMSE values reported give an indication that the proposed method can generate similarly low-RMSE predictions.

One of the biggest benefits of using proposed approach to ship motion prediction is its reduced computational time. In Zhao's comparative study it was shown that the MCA algorithm took $40.9 \mathrm{~s}$ to train and predict using $400 \mathrm{~s}$ of original data and making a prediction $20 \mathrm{~s}$ [7]. In the same study, the AR algorithm, Wiener prediction, and the neural network mentioned before took $53.4 \mathrm{~s}, 1054 \mathrm{~s}$, and $3470 \mathrm{~s}$ respectively to train and predict.

For a dataset with a $50 \mathrm{~s}$ sampling interval, the proposed method produces computational times as shown in Fig. 12 using the start-up procedure. Using a $400 \mathrm{~s}$ sampling interval, matching that used in the MCA algorithm used by Zhao, the proposed algorithm takes $4.39 \mathrm{~s}$ to fit 15 sine waves and make a $20 \mathrm{~s}$ prediction.

Figure 13 shows the computational time for the realtime application compared to the time taken for the start-

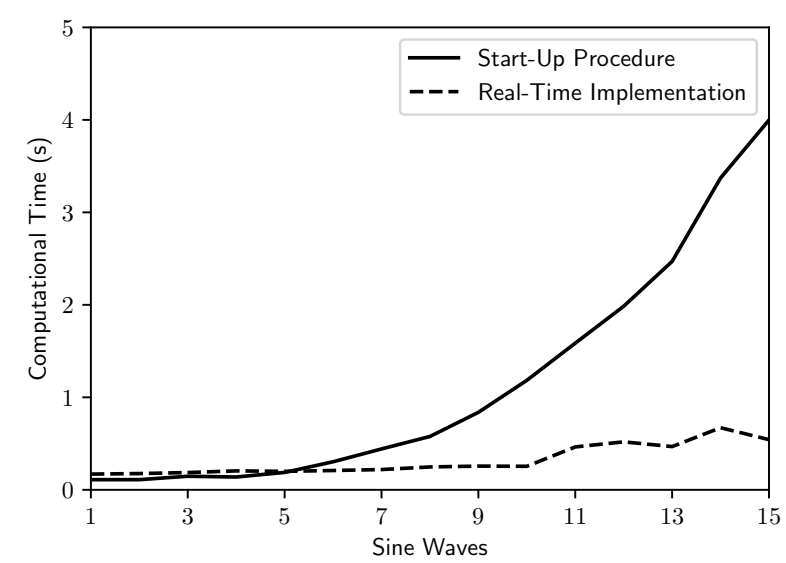

Fig. 13. Computation time for the real-time and start-up procedures of a $50 \mathrm{~s}$ sampling interval of original data.

up procedure. Both the real-time and start-up scenarios were performed over a $50 \mathrm{~s}$ time interval. These computational times were found using an Intel Core i7-4790 CPU with a processing speed of $3.60 \mathrm{GHz}$. When using the real-time application, the computational times are drastically reduced when compared to the start-up procedure and show improved prediction times when compared to the other methods.

The RMSE of the subsequent real-time updates do not vary significantly from those reported by the initial iteration when applied over a short period of time. It is important to emphasize that these computational times could be reduced by an order of magnitude if written in a compiled language. Python was used for readability and ease-of-use, but it requires significantly more time to perform the calculations in the proposed algorithm than a compiled language.

Information for the computational time of other methods was scarce. However, the results of the sine wave summation algorithm are promising, and indicate that computational time of the algorithm is low, and therefore well-suited to making timely deck motion predictions for pilots.

\section{CONClusion}

Landing a fixed-wing aircraft on an aircraft carrier is a challenging process for pilots. This challenge has necessitated research into accurate ship motion prediction. An overview of the physics of ship motion was used to give context to the proposed sine-summation prediction method. It was shown that the motion of a ship subjected to the forcing function produced by ocean waves is a simple summation of sine waves.

The sine-summation method was shown to have two components, a start-up procedure and a real-time algorithm. The start-up procedure utilized an FFT to extract harmonic properties from ship motion data. A sine wave that characterized a portion of the dataset was then created using the harmonic parameters found by the FFT. A gradient-descent optimization was performed, which minimized the RMSE between the sine wave and the original dataset. This process 
was repeated until a summation of sine waves had been created to approximate the signal. The final summation was projected into the future as a prediction of future ship motion that could be compared to actual future data.

The real-time application of the method was also reported, which maintained the RMSE benefits of the iterative approach while requiring less computational time than the startup procedure. An up-front computational cost was required by the real-time application in the form of the start-up procedure, but represents the method most likely to be used on-board an aircraft carrier.

Additional research into the application of the proposed method could help to increase the accuracy of the predictions. The iterative solution proposed in this work is not necessarily the most efficient way to extract the harmonic components present in a given dataset. Further analysis into the most efficient way to extract these components could remove the need to perform the minimization of the RMSE during the start-up procedure.

In addition, there seems to be a significant correlation between the accuracy of the prediction and the dataset being analyzed. Ultimately, the proposed method would need to be robust across a large range of datasets. A great deal of research could be done to further understand the correlation between RMSE and the dataset used and adapt the algorithm to further increase the accuracy of the prediction. Finally, the results outlined in Section IV are in no way comprehensive, and could be expanded to understand the interactions between sampling interval, the number of sine waves in a fit, and the prediction time interval with regards to prediction RMSE.

The proposed sine-summation algorithm was shown to be comparable to the other methods currently being used to predict ship motion. The algorithm has a low computational complexity and produces RMSE values that are on the same order of magnitude as those presented in other methods. In addition, the real-time capabilities of the proposed algorithm allow an even greater decrease in computational time. As it stands, the concept of sine-summation prediction method has been verified as a potential solution to the ship motion prediction problem.

\section{ACKNOWLEDGMENT}

This research was made possible by the Utah State University Engineering Undergraduate Research Program (EURP). EURP receives its funding from the Mechanical and Aerospace Engineering Department of the College of Engineering at Utah State University and the USU Aerolab. Acknowledgment is also given to ONR and the Naval Surface Warfare Center Caderock Division (NSWCCD) for access to the SCONE dataset. Those interested in obtaining the dataset should contact Alan Schwartz, NSWCCD Code 882, via email at alan.schwartz@navy.mil.

\section{REFERENCES}

[1] R. A. Hess and . M. Judd, "Improved automatic carrier landing using deck motion prediction," Journal of Aircraft, vol. 13, no. 2, pp. 153-155, Feb. 1976. [Online]. Available: http://arc.aiaa.org/doi/10.2514/3.44514

[2] N. Sandlin, R. Ebers, and R. Black, "Ship motion effects on landing impact loadsV/STOL landing on aircraft carrier." American Institute of Aeronautics and Astronautics, Apr. 1979. [Online]. Available: http://arc.aiaa.org/doi/10.2514/6.1979-742

[3] S. Chakrabarti, Handbook of Offshore Engineering (2-volume set). Elsevier, 2005.

[4] S. Küchler, T. Mahl, J. Neupert, K. Schneider, and O. Sawodny, "Active control for an offshore crane using prediction of the vessel's motion," IEEE/ASME Transactions on Mechatronics, vol. 16, no. 2, pp. 297-309, 2011.

[5] J. Falnes, Ocean Waves and Oscillating Systems: Linear Interactions Including Wave-Energy Extraction, 1st ed. Norwegian University of Science and Technology, Trondheim: Cambridge University Press, Apr. 2002.

[6] J. Journee and J. Pinkster, "Introduction in ship hydromechanics," Delft University of Technology, p. 8, 2002.

[7] X. Zhao, R. Xu, and C. Kwan, "Ship-motion prediction: algorithms and simulation results," in Acoustics, Speech, and Signal Processing, 2004. Proceedings.(ICASSP'04). IEEE International Conference on, vol. 5. IEEE, 2004, pp. V-125.

[8] L. Keke, C. Nong, and L. Qing, "Research and simulation on the carrier deck motion adaptive prediction for ACLS design," in Guidance, Navigation and Control Conference (CGNCC), 2014 IEEE Chinese. IEEE, 2014, pp. 1341-1345.

[9] I. R. Yumori, "Real time prediction of ship response to ocean waves using time series analysis," in OCEANS 81. IEEE, 1981, pp. 10821089.

[10] P. Xiuyan, Z. Biao, and R. Lihong, "Ship motion prediction of combination forecasting model based on adaptive variable weight," in 2015 34th Chinese Control Conference (CCC), Jul. 2015, pp. 40154019.

[11] M. Sidar and B. Doolin, "On the feasibility of real-time prediction of aircraft carrier motion at sea," IEEE Transactions on Automatic Control, vol. 28, no. 3, pp. 350-356, 1983.

[12] X. Yang, H. Pota, M. Garratt, and V. Ugrinovskii, "Ship Motion Prediction for Maritime Flight Operations," IFAC Proceedings Volumes, vol. 41, no. 2, pp. 12 407-12 412, 2008.

[13] P. Kaplan, "A Study of Prediction Techniques for Aircraft Carrier Motions at Sea," Journal of Hydronautics, vol. 3, no. 3, pp. 121-131, 1969. [Online]. Available: https://doi.org/10.2514/3.62814

[14] J. F. Horn, J. Yang, C. He, D. Lee, and J. K. Tritschler, "Autonomous ship approach and landing using dynamic inversion control with deck motion prediction," 2015.

[15] F.-L. Luo, R. Unbehauen, and A. Cichocki, "A Minor Component Analysis Algorithm,” Neural Networks, vol. 10, no. 2, pp. 291-297, Mar. 1997.

[16] D. Peng and Z. Yi, "A new algorithm for sequential minor component analysis," International Journal of Computational Intelligence Research, 2006.

[17] X. Liu, Q. Wang, Y. Huang, Q. Song, and L. Zhao, "A prediction method for deck motion of aircraft carrier based on particle swarm optimization and kernel extreme learning machine," Sens Mater, vol. 29, no. 9, pp. 1291-1303, 2017.

[18] J.-C. Yin, Z.-J. Zou, F. Xu, and N.-N. Wang, "Online ship roll motion prediction based on grey sequential extreme learning machine," Neurocomputing, vol. 129, pp. 168-174, 2014.

[19] W. Zhang and Z. Liu, "Real-time ship motion prediction based on time delay wavelet neural network," Journal of Applied Mathematics, vol. 2014, 2014.

[20] C. Eckart, "Internal waves in the ocean," The Physics of Fluids, vol. 4, no. 7, pp. 791-799, 1961.

[21] S. L. Marple Jr., Digital Spectral Analysis with Applications, ser. Prentice-Hall Signal Processing Series. New Jersey: Prentice-Hall, Inc., 1987.

[22] C. G. Broyden, "The convergence of a class of double-rank minimization algorithms 1. general considerations," IMA Journal of Applied Mathematics, vol. 6, no. 1, pp. 76-90, 1970.

[23] R. Fletcher, "A new approach to variable metric algorithms," The computer journal, vol. 13, no. 3, pp. 317-322, 1970.

[24] D. Goldfarb, "A family of variable-metric methods derived by variational means," Mathematics of computation, vol. 24, no. 109, pp. 23-26, 1970. 
[25] D. F. Shanno, "Conditioning of quasi-newton methods for function minimization," Mathematics of computation, vol. 24, no. 111, pp. 647656, 1970.

[26] J. A. Nelder and R. Mead, "A simplex method for function minimization," The computer journal, vol. 7, no. 4, pp. 308-313, 1965.

[27] A. Schwartz, "Systematic characterization of the naval environment (scone) - standard deck motion data for a generic surface combatant," 2015. [Online]. Available: http://www.navalengineers.org/Symposia/PastSymposia/Launch-and-Recovery-2016/Program/Schwartz

[28] A. Chinn, "LAMP: Large Amplitude Motion Program," NRC Institute for Ocean Technology; National Research Council Canada, St. John's, NL, Technical Report 6017, 2004. [Online]. Available: http://nparc.nrc-cnrc.gc.ca/eng/view/object/?id=86a32151e965-4e98-81e4-6bd098a0fc4b

[29] T. Basar and I. S. IEEE, Control Theory: Twenty-Five Seminal Papers (1932-1981). IEEE Press New York, 2001.

[30] W. J. Rugh, Nonlinear system theory. Johns Hopkins University Press Baltimore, 1981 